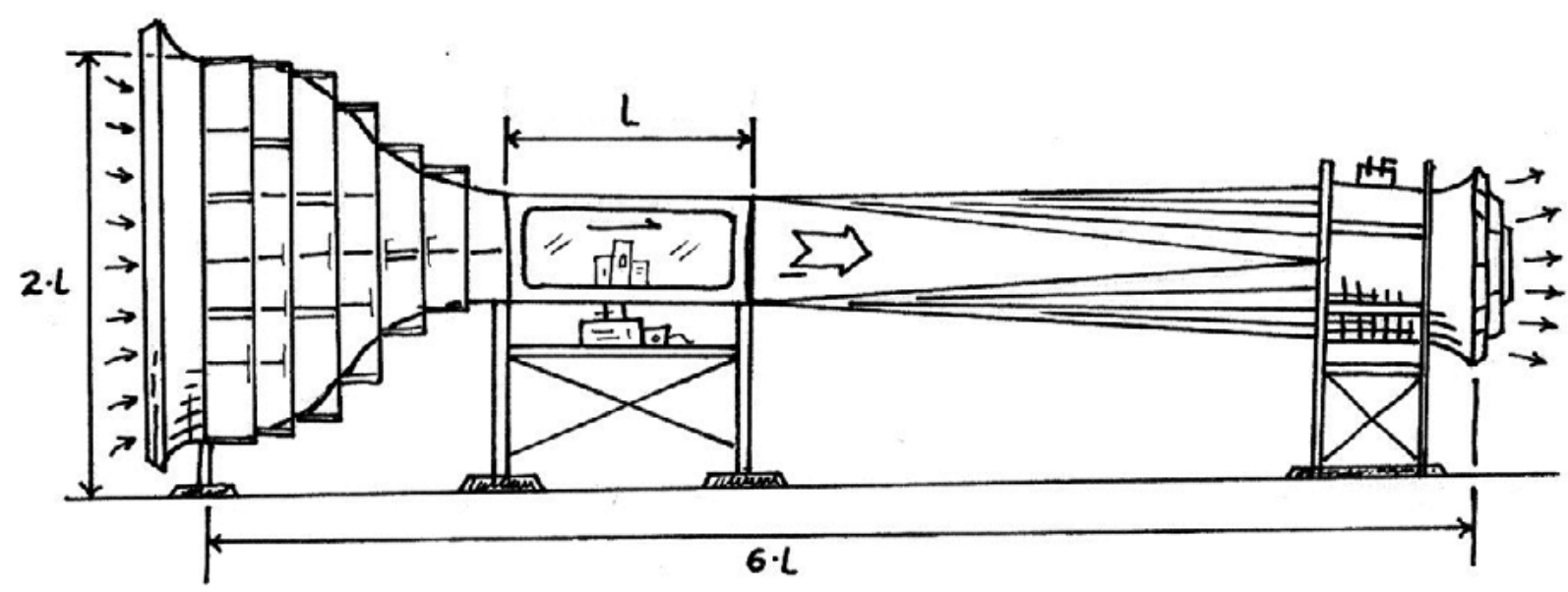

\title{
CONCEPTO PARA UN TÚNEL DE VIENTO CON VÓRTICE VARIABLE
}

\section{CONCEPT FOR A VARIABLE VORTEX WIND TUNNEL}

\section{Fecha de recepción: 09 de Octubre de 2015}

Ing. Juan Gabriel Monge-Gapper, M.Sc

Director del Posgrado en Ingeniería Mecánica

Universidad de Costa Rica, Costa Rica

E-mail: juan.mongegapper@ucr.ac.cr

\section{RESUMEN}

Se propone un diseño de bajo costo de un túnel de viento con vórtice variable, que mediante la inyección controlada de flujos cruzados direccionales, permite variar el tamaño y velocidad de rotación de los vórtices independientemente de la velocidad promedio del flujo. Se evita con ello la reconfiguración de la sección de estabilización, comúnmente utilizado en los túneles de capa limite. El uso de este túnel de viento en la escala y velocidad indicada serían para pruebas prácticas usando modelos a escala para cuantificar la interacción del viento atmosférico con construcciones civiles, caracterizar patrones de erosión de terrenos y transporte de sedimentos, y determinar los patrones generales de dispersión de contaminantes atmosféricos en ciertas escalas de terreno; podría utilizarse también para definir parámetros de calibración en modelos numéricos de flujo de fluidos a baja velocidad en dos dimensiones.

\section{PALABRAS CLAVE:}

Túnel de viento, capa límite atmosférica, flujo rotacional, ingeniería del viento.

\section{ABSTRACT}

This paper describes the basic design of a low-cost boundary layer wind tunnel specially conceived to be much shorter than conventional environmental wind tunnels while allowing for real-time adjustment of vortex size and speed without needing to alter mean flow speed or affecting fan performance. Potential applications include medium scale modeling of atmospheric wind as it interacts with large urban areas, soil erosion, sediment transportation, and large-area pollution dispersion. Also, calibration of some numerical models of two dimensional low-speed fluid flow may be accomplished with adequate instrumentation.

\section{KEYWORDS:}

Wind tunnel, atmospheric boundary layer, rotational flow, wind engineering. 


\section{INTRODUCCIÓN}

Los túneles de viento de capa límite atmosférica se desarrollaron para modelar a escala reducida el efecto que tendría el viento atmosférico en construcciones o emplazamientos de determinado interés práctico (Barlow et al, 1999). Dada la variabilidad de la velocidad del viento en función de la distancia con la superficie, su vorticidad, y los cambios que presenta en un sitio real, la reproducción en un túnel de viento demanda una serie de complicaciones técnicas adicionales a los que convencionalmente están presentes en un túnel de viento subsónico.

Sólo el hecho de reproducir un perfil de distribución de velocidad con sesgo parabólico respecto de una de las paredes del túnel (que corresponde al suelo del emplazamiento) obliga a introducir un largo ducto de preparación del caudal (Schlichting, 2000) con paredes dotadas de elementos de fricción diseñados a la medida de la curvatura de la parábola esperada y del grado de turbulencia requerido (Bianco et al, 2008; Kundu y Cohen 2015).

Convencionalmente, los túneles de viento se diseñan y construyen con cuidada atención a minimizar la turbulencia presente en la sección de pruebas (Okamoto, 2011); en un túnel de viento de capa límite el grado de turbulencia más bien debe llevarse al nivel análogo al que ocurre en las condiciones de viento atmosférico, teniendo en cuenta los efectos de escala que puedan afectar en medida significativa las mediciones al objeto de pruebas.

Si además del perfil de velocidades y del grado de turbulencia se desea modelar el efecto de diferenciales de temperatura, presencia de precipitación, o la periodicidad de las ráfagas, la complejidad técnica del túnel de viento junto con su equipo de control y los sistemas de medición se vuelve considerablemente costoso.

La duración de las pruebas en túnel de viento y el costo asociado hace que la estimación de fuerzas en una estructura debidas a viento atmosférico se acostumbre hacer con lo documentado en códigos de diseño, los cuales se fundamentan en la clasificación por máxima velocidad de viento local (CFIA, 2010), y se pueden complementar con factores de forma experimentales (ASCE 2013). Si el caso lo permite, se puede complementar este cálculo con modelos numéricos preparados con paquetes de cómputo comerciales cuyo margen de error es razonablemente bajo (Hirsch 2007), especialmente considerando que la mayoría de diseñadores estudiará cargas de viento críticas para el emplazamiento de una estructura.

Una de las desventajas de diseño por código es que, aunque es razonablemente seguro, no provee visión del fenómeno de mecánica de fluidos ni señala dirección de optimización de la estructura; tampoco provee forma para obtener las frecuencias de resonancia debidas al flujo.
Un modelo numérico es un excelente complemento, dado que puede proveer buena aproximación de la distribución de presiones y velocidades que puedan ocurrir en un determinado emplazamiento. Dado que se trata de un modelo computacional, la modificación de alguna característica geométrica es relativamente sencilla en comparación con llevar a cabo mediciones en una construcción a escala real o en una maqueta en un túnel de viento.

Cuando haya fenómenos de viento cuyo efecto no pueda determinarse con los modelos numéricos por limitaciones técnicas o de capacidad, entonce:; es justificable recurrir a pruebas en túnel de viento, pero el acceso es limitado, y por ende los costos son cada vez ma rores. Muchos túneles de viento construidos entre 1920 y 1990 han cerrado operaciones (USAF, 1990) dado su alto costo de operación y bajo factor de uso, especialmente a la luz de la conveniencia y precisión que proveen los modelos numéricos disponibles en paquetes de cómputo comerciales después del año 2005 (Hirsch, 2007).

Dicho esto, el túnel de viento está lejos de ser innecesario; aún hay muchos fenómenos que no pueden reproducirse convenientemente con métodos numéricos, al punto que a la fecha no han dejado de construirse túneles de viento para modelar condiciones de flujo especiales y obtener datos acerca de fenómenos de mecánica de fluidos a toda escala. Un caso de especial interés para el estudio del efecto de vientos huracanados en zonas habitadas es el túnel de viento hexagonal del Instituto de Investigaciones en Ingeniería del Viento, Energía y Ambiente (Wind Engineering, Energy and Environment Research Institute) de la Universidad del Oeste de Ontario (University of Western Ontario). Esta obra inició operaciones en Canadá en el año 2013 (Hangan, 2015) y su geometría y configuración permite modelar en extensiones considerables de topografía a escala reducida viento atmosférico en combinación con succión (tornados) y descarga (huracanes) perpendiculares al suelo (Roberts, 2012).

El concepto de un túnel de viento de vórtice variable surgió como una versión modificada de un túnel de viento de capa límite cuyo propósito era que se pudiera acortar la sección de preparación (Monge, 2010), (Shojaee, 2014) que es característica de estos equipos.

En este artículo se presenta un concepto de equipo para la determinación de cargas de viento y caracterización de condiciones de flujo. En vista de que este tipo de instrumento ocupa una cantidad considerable de espacio físico y es complejo de operar para obtener mediciones pertinentes de carga de viento en modelos estructurales, este documento se ha generado para difundir las potenciales aplicaciones y la función prevista de un túnel de viento que no sólo puede cubrir aplicaciones generales como en otros centros de investigación de clase internacional, sino que además incorpora la novedad de un generador de vórtice variable. 


\section{CONFIGURACIÓN GENERAL}

Tanto diseñadores como usuarios de un túnel de viento coinciden históricamente que un túnel de viento debe hacerse tan grande como lo permita el presupuesto y el espacio físico disponible (Barlow et al, 1999) dado el impacto directo que tiene en los factores de escala necesarios para los modelos de estudio. Entre mayor sea la sección transversal del tramo de pruebas, menor será la interferencia de las paredes (Bernstein, 1976) y otros efectos de escala y será más sencilla la instalación de instrumentos para medir de velocidad y presión local en el modelo.

Esta condición resulta en que haya una amplia variedad de diseños y tamaños de túnel de viento, en donde las aplicaciones potenciales demandan soluciones creativas para obtener al menor costo la condición de flujo necesaria para los experimentos que deseen desarrollarse.

En este caso en particular, lo que se persigue es un túnel de viento el que se puedan ensayar modelos de edificaciones a escalas entre 1:50 y 1:50 y terrenos a escalas de 1:750 a 1:5000, principalmente para efectos de observar patrones de flujo y determinar frecuencias de excitación en modelos rígidos. A estas escalas, las maquetas resultan muy grandes para que puedan introducirse en los túneles de viento didácticos disponibles en el país, que tienen una sección de pruebas de $300 \mathrm{~mm}$ x $300 \mathrm{~mm}$ y no desarrollan una capa límite con el perfil de velocidades propio del viento atmosférico (Rivera, 2005).

La demanda inicial para un túnel de viento en el contexto regional será para pro pósitos didácticos y de demostración, por lo que se espera una inversión inicial baja que pueda complementarse con mejor instrumentación conforme haya disponibilidad presupuestaria.

El punto de partida del diseño geométrico es una configuración abierta convencional tipo Eiffel como el de la Figura 1. Para esta configuración, las proporciones y métodos de construcción se encuentran documentados para muchos tamaños (Barlow et al, 1999), pero eso obliga a que para velocidades cercanas a $90 \mathrm{~m} / \mathrm{s}(325 \mathrm{~km} / \mathrm{h})$ en la sección de pruebas el ensamble completo tendrá una longitud de al menos seis veces la longitud de la sección de pruebas, lo que encarece considerablemente la construcción y su adecuada protección contra la intemperie o de distorsiones ambientales que afecten las mediciones.

Si la sección de pruebas se elige demasiado pequeña, no se presta para modelar el flujo de masas de aire como el que ocurre en la atmósfera a debajo de los $700 \mathrm{~m}$ sobre la superficie (Bianco et al, 2008). Por otro lado, una sección de trabajo grande también queda asociada a ventiladores que demandan altas potencias, del orden de $100 \mathrm{~kW}$ cuando la velocidad efectiva es de unos $25 \mathrm{~m} / \mathrm{s}(90 \mathrm{~km} / \mathrm{h}$ ) (Mehta y Bradshaw, 1979) y los niveles de ruido no son aceptables para operarse cerca de localidades habitadas (Knight, 2001).

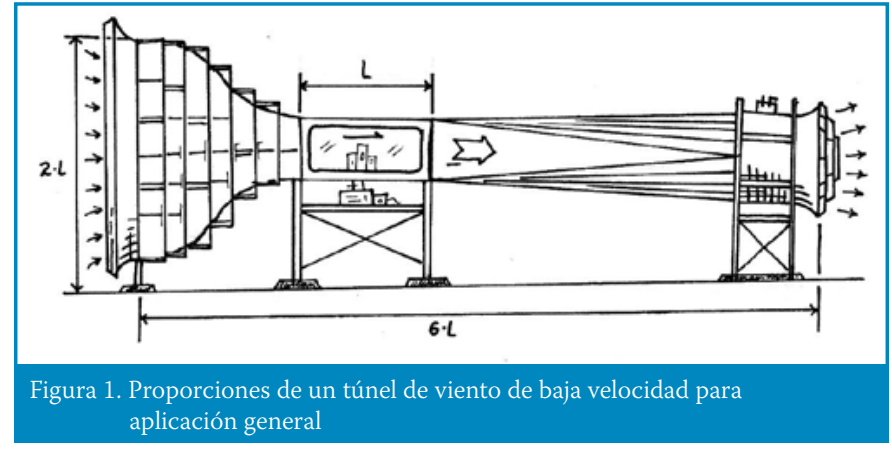

Estas proporciones serían necesarias para cubrir un ámbito relativamente amplio de velocidades subsónicas, pero conforme es menor la velocidad máxima de operación, la campana de contracción no necesita ser tan extrema. Para modelar viento atmosférico en maquetas con un factor de escala no muy alto, si se dispone de instrumentación adecuada no son necesarias velocidades superiores a los 15 $\mathrm{m} / \mathrm{s}$ (54 km/h) (Pegg, 1995), con la ventaja adicional de que se reducirá considerablemente su costo y los niveles de ruido del abanico.

Se propone la configuración Eiffel modificada para las condiciones especiales de reproducir a escala el perfil de velocidades propio de la capa límite atmosférica para una sección de trabajo de $1200 \mathrm{~mm}$ x $800 \mathrm{~mm}$. La geometría general correspondiente se muestra en la Figura 2.

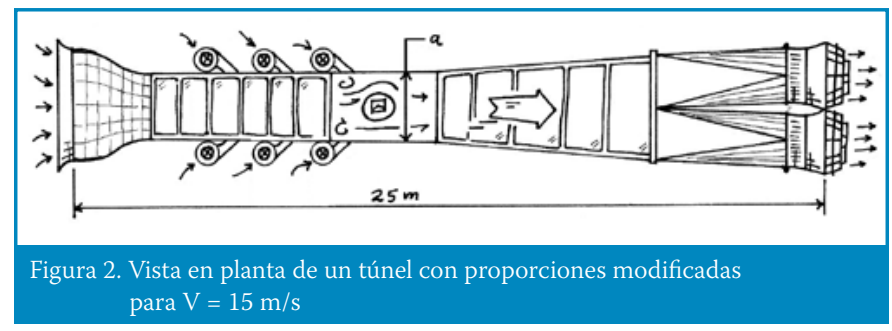

Las modificaciones más importantes a las proporciones de la ductería son la campana de contracción pequeña y la presencia de los dos abanicos principales en lugar de uno solo al término del ducto; eso acorta considerablemente el difusor. La campana de contracción se optimiza para la velocidad máxima de $15 \mathrm{~m} / \mathrm{s}$, para evitar la relación de área de succión a descarga alta (Barlow et al, 1999) que caracteriza los túneles de viento para aplicación general.

Como es evidente, buena parte de la longitud total del túnel es ductería, ya sea como transición suave entre la succión del ventilador y la descarga de la sección de trabajo o el tramo agregado antes de la sección de pruebas (tramo a de la Figura 2) que prepara el perfil de viento atmosférico. Esta zona, es donde se colocan los abanicos secundarios para generación activa y variable de vórtices como se muestra en la Figura 3. Esto afecta el espacio físico que cubre, así como el tamaño, potencia y tipo de los abanicos principales que mueven el flujo de aire. 


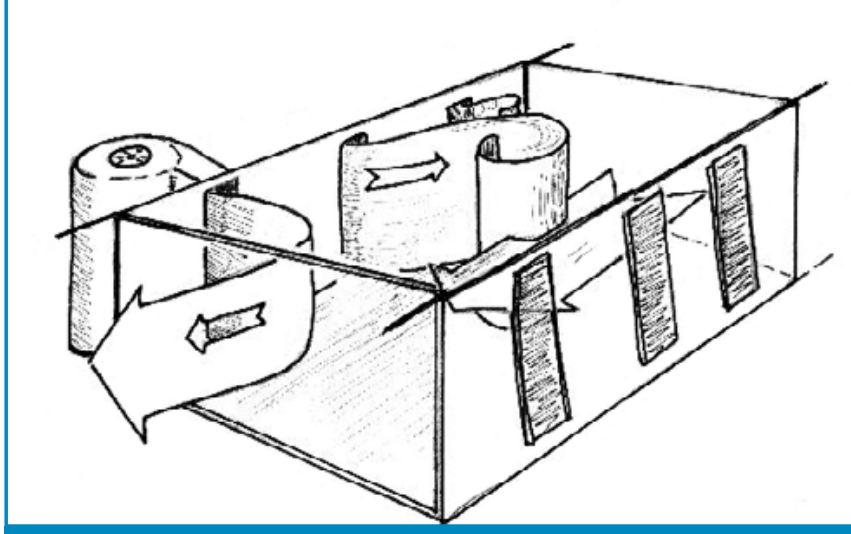

Figura 3. Croquis de la sección con inyección de flujo para generación de vórtices

Los abanicos principales que se colocan al término de la ductería proveen el flujo dominante, y son de tipo axial; aparte de ello, los dos aspectos más importantes en su selección son los niveles de ruido y la potencia requerida dado que afectan directamente el costo del sistema.

La instrumentación y servicios asociados a un túnel de viento no son únicos, ni tienen que formar parte permanente de él, pero son indispensables para las actividades que desarrollen docentes e investigadores que puedan aprovechar un flujo de aire en condiciones controladas.

\section{APLICACIONES INMEDIATAS}

El propósito de un túnel de viento especialmente diseñado para visualizar y cuantificar el efecto del viento atmosférico en estructuras y terrenos se puede asociar a investigaciones en muchas áreas del conocimiento activas a nivel local. En este caso se tendría la ventaja adicional de que se puede variar el grado de vorticidad independiente de la velocidad media de flujo; en la mayoría de túneles de viento de capa límite, la turbulencia es proporcional a la velocidad.

Hasta la fecha, únicamente los túneles de viento de capa límite con techo o suelo móvil pueden variar estos parámetros con alguna independencia (Pereira, 2013), y requieren complejos sistemas para variar la posición de los diferentes tramos de ducto. El túnel de viento de vórtice variable permite variar la vorticidad sin necesidad de los costosos sistemas hidráulicos y ductería de construcción especial. Además, puede hacerlo en el transcurso de un experimento, lo que no es practicable en túneles de paredes móviles.

Asumiendo que se encuentra disponible la instrumentación básica necesaria, el tamaño, velocidad y uniformidad del diseño propuesto permitirían los siguientes tipos de pruebas, casi siempre sobre modelos geométricos precisos de la zona de interés:

Patrones de movimiento de viento cerca de accidentes geográficos. Por medio de imágenes, hilos de humo y sistemas de procesamiento de imágenes afines, se obtiene una perspectiva de las líneas de flujo y zonas con flujo rotacional o estancado en las diferentes áreas que incluya el modelo físico. Las mediciones directas de velocidad y presión por lo general no son muy significativas dado que los efectos de escala afectan mucho la precisión de los resultados. Los factores de escala usuales se encuentran entre 1:750 y 1:5000 (Reinholt, 1982).

Campos de velocidades y presiones del viento. Cuando se trate de modelos de edificios y de estructuras y otras construcciones con factores de escala entre $1: 50$ y 1:150, se pueden hacer mediciones directas de velocidad y de presión directamente en la superficie del modelo o en zonas alejadas de las paredes del túnel de viento sin que se pierda precisión por interferencia del instrumento de medición (Reinholt, 1982). Con los factores de corrección adecuados se pueden extrapolar los resultados a un modelo a escala natural con precisión suficiente para tomar decisiones de ingeniería. Las líneas de flujo se pueden hacer visibles con métodos de túnel de viento probados (Barlow et al, 1999).

Patrones de erosión y transporte en terrenos. Si se elige un material con la cohesividad, densidad y rugosidad superficial adecuadas, se puede observar la acción directa del viento atmosférico en modelos a escala reducida de terrenos susceptibles a la erosión (Shao, 2008). Esto es de especial utilidad para modelos de terrenos irregulares en donde se necesita conocer el efecto de la topografía, la vegetación y las barreras en los patrones y magnitud relativa de los procesos de erosión por viento. Necesitan complementarse con otros modelos fuera del túnel que agreguen la componente de las precipitaciones o de la erosión hidráulica.

Patrones de arrastre de inundaciones por efecto del viento. Para ciertos factores de escala función del espesor de la capa de agua, se puede determinar la dirección y patrón general de movimiento de agua arrastrada por efecto del viento atmosférico en modelos limitados de zonas costeras (Shao, 2008). Estos modelos son difíciles de calibrar para factores de escala altos y por lo general son de interés didáctico para ciertas configuraciones conocidas.

Patrones de daño a vegetación en terrenos irregulares. Por medio de modelos de terrenos con vegetación, se puede determinar por medición directa de velocidad el daño probable a la vegetación por áreas y por tipo de flujo (zonas estancadas, de alta turbulencia, o de altas velocidades por efecto Venturi). Entre menor sea el factor de escala, más precisos serán los resultados, y eso a su vez depende del tamaño real de la vegetación que se necesita valorar.

Visualización de patrones de dispersión de contaminantes. En el modelo de un emplazamiento se puede liberar un marcador gaseoso o una suspensión de partículas para determinar la dirección, distribución y grado de dispersión que tendría. La precisión del modelo es función de la densidad media y de la difusividad a escala del marcador (Ahmad et al, 2005) en proporción a las relaciones que se darían a escala natural con el contaminante que se desea modelar. 
Experimentoscualitativosderesonanciaporretroalimentación. La interacción entre el viento atmosférico y una estructura elástica es un fenómeno complejo por cuanto es necesario que la elasticidad del modelo lleve una proporción muy precisa; además, a escala natural la afectan muchos factores como la variabilidad de la velocidad del viento, vorticidad irregular y otras cargas activas que no dependen del viento. Por ende, la construcción de modelos es muy costosa y de utilidad limitada a una aplicación muy particular, pero es uno de los pocos campos en que los modelos numéricos aún no producen resultados suficientemente precisos (Anina et al, 2015) dado que la frecuencia y modo de resonancia del fluido (estrictamente el fluido no resuena, sino que exhibe patrones de turbulencia en fase con la deformación de la estructura) la afecta la deformación periódica del sólido elástico, que a su vez recibe una carga variable porque su orientación respecto al fluido cambia la carga que el fluido ejerce.

Demostraciones y experimentos clásicos en mecánica de fluidos. Si bien muchos experimentos y demostraciones de mecánica de fluidos se facilitan si se llevan a cabo en un túnel de viento de menor tamaño, hay algunas experiencias que serán más valiosas en un túnel de viento con una sección transversal de mayor tamaño, aún cuando la velocidad de flujo sea considerablemente menor. Cuando se necesite visualizar flujo potencial tridimensional, o si se necesita generar un perfil de velocidades y presiones en un objeto de geometría compleja, la sección transversal ayuda a que las mediciones resulten más repetibles y consecuentes con resultados de otras fuentes, de modelos numéricos o planteos teóricos.

Muchas de estas pruebas no responden a un área del conocimiento única, y rara vez podría dedicarse el equipo a un solo tipo de prueba; son esencialmente transdisciplinarias en vista de que combinan la mecánica de los fluidos con la determinación de los efectos que tiene sobre un sistema, que puede ser elástico, particulado, sólido, o sencillamente un estudio geométrico. Esta misma configuración de túnel de viento permitiría además pruebas relacionadas con la determinación de fuerzas y turbulencias locales en construcciones civiles, verificación de sistemas con ventilación natural u obtención de velocidades de flujo en áreas de tránsito peatonal o vehicular.

Por otra parte, hay una gran cantidad de actividades regulares de docencia o de difusión general que pueden aprovechar un flujo constante de aire en condiciones controladas de velocidad y vorticidad, siempre que se disponga de los instrumentos de medición pertinentes al tipo de prueba que se desea ejecutar.

\section{DIMENSIONES DE LA SECCIÓN DE PRUEBAS}

Cada una de las secciones tiene dimensiones y proporciones en función del tamaño de la sección de prueba y de la velocidad de trabajo requerida, usualmente debajo de los $12 \mathrm{~m} / \mathrm{s}(45 \mathrm{~km} / \mathrm{h})$ para las aplicaciones mencionadas en la sección anterior. Se propone una estructura que combina la durabilidad y facilidad de construcción del perfil de acero con el bajo costo y capacidad de absorber vibraciones de la madera contrachapada. El uso de madera en las paredes facilita las reparaciones o la reposición de tramos que se dañen o necesiten modificarse temporalmente para recibir modelos o instrumentación especial.

La sección de trabajo define el tamaño del resto de la ductería y, junto con el ámbito de velocidades seleccionado, la potencia requerida de los abanicos. Un ancho de $1200 \mathrm{~mm}$ es suficiente para que las maquetas de los terrenos puedan construirse en factores de escala de entre 1:750 y 1:5000 (Reinholt, 1982) sin excederse en las imprecisiones propias del uso de modelos a escala. La altura de $800 \mathrm{~mm}$ se escogió con fundamento en la proporción 3:4, muy frecuente en el diseño de túneles de viento de capa límite (Barlow et al, 1999; Goodrich y Gorham, 2008) para dar una altura libre suficiente para evitar efectos de pared.

La contracción es la sección de más compleja construcción, en vista de que está compuesta por tramos curvos cuya curvatura debe carecer de discontinuidades para no generar separación de flujo antes de que inicie el tramo de preparación de capa límite (Mehta y Bradshaw, 1979). Su propósito es acelerar suavemente el aire exterior a una condición de flujo de velocidad constante en la descarga. Por lo general la entrada dispone de varias mallas de alambre consecutivas para reducir la influencia de corrientes de aire que tienden a distorsionar el flujo.

El generador de capa límite es un tramo de ducto cuyas paredes interiores tienen irregularidades especialmente dispuestas para que a su descarga el perfil de velocidades sea aproximadamente parabólica (Shojaee et al, 2014) pero con cierto grado de vorticidad, semejante al del viento atmosférico cercano al suelo (conocido como perfil de capa límite atmosférica para elevaciones inferiores a los $700 \mathrm{~m}$ ). Este tramo podría ser removible para el caso de pruebas que deseen hacerse con un perfil de velocidades homogéneo para experimentos de calibración de modelos teóricos o demostraciones.

Esta sección merece especial atención por cuanto es en donde se incorporan seis abanicos centrífugos tipo cortina cuya descarga puede orientarse según se muestra en la Figura 4 para producir diferentes grados de interacción cortante con el flujo principal. Esto provocará, según la relación de velocidades y el ángulo de descarga, distintas velocidades 
de rotación en columnas verticales aledañas a las paredes interiores de la sección de pruebas.

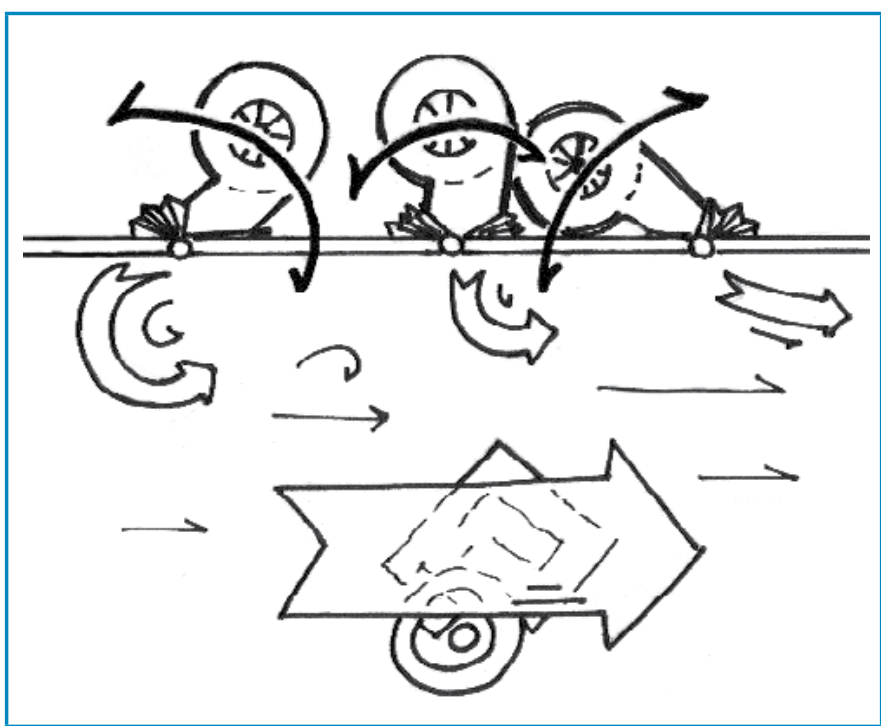

Figura 4. Efectos de los ventiladores orientables para la generar de columnas rotacionales

Si los ventiladores disponen de motores de velocidad variable, se tendrá la ventaja adicional de que se podrían alterar tres diferentes parámetros de flujo para provocar una amplia variedad de combinaciones de velocidad media de viento y grado de vorticidad.

El difusor cumple la función de transición entre la forma rectangular de la salida de la sección de pruebas a la sección redonda propia de la entrada a los abanicos de flujo axial. Su longitud considerable se debe tanto a que el ángulo del difusor no puede ser mayor a unos $2^{\circ}$ (Barlow et al, 1999; Kundu y Cohen, 2015; Mehta y Bradshaw, 1979) y a que los ventiladores principales deben recibir un flujo razonablemente estable para que no se pierda calidad del flujo.

El área considerable que esta ductería demanda se verá aumentada por las zonas libres que deben dejarse en la succión y a la descarga; como es evidente, esto resulta en una planta ocupada de al menos $25 \mathrm{~m} \mathrm{x} 5 \mathrm{~m}$. Idealmente, el túnel se debería encontrar a salvo de la intemperie para que el viento atmosférico -verdadero-no altere los experimentos. Un encierro también reduce el impacto ambiental del ruido de los abanicos.

\section{VENTILADORES PARA IMPULSO Y VÓRTICE VARIABLE}

El alto costo inicial de un túnel de viento debe buena parte a los motores, abanicos y el sistema de control de velocidad. La potencia consumida (lo que afecta directamente el precio de los abanicos y del sistema de control de velocidad) crece en proporción al cuadrado de la velocidad de flujo, por lo que los costos aumentan rápidamente al elegir velocidades de flujo mayores. Es por ello que no es extraño encontrar velocidades máximas tan bajas como $8 \mathrm{~m} / \mathrm{s}$ (unos $25 \mathrm{~km} / \mathrm{h}$ ) en los túneles de viento de capa límite (Pereira, 2013); en este caso en particular, se elige que el flujo principal se impulse con dos abanicos de común uso en la industria para evitar el costo que implica obtener y operar abanicos diseñados a la medida.

La selección de los abanicos y del sistema de control es un tema que ha generado mucha discusión y documentación pública en forma de artículos científicos y ponencias, pero no hay una solución única universal dada la variedad de aplicaciones que puede tener un túnel de viento. Para túneles de viento de baja velocidad tipo Eiffel, una opción atractiva en términos de costo inicial y control preciso de la velocidad es el uso de abanicos de flujo axial de aspas fijas impulsado por un motor eléctrico de corriente alterna con un variador de frecuencia (Goodrich y Gorham, 2008). El variador de frecuencia faculta un ámbito de velocidades muy amplio (Chapman, 2010) con la posibilidad de que reciba retroalimentación de un centro de control para mantener el caudal constante, lo que reduce la incertidumbre por temperatura del aire, cambios en el rendimiento del abanico, o variaciones del voltaje de línea.

Para la sección transversal propuesta de $1200 \mathrm{~mm} \times 800$ $\mathrm{mm}$ y una velocidad en la sección de pruebas de $15 \mathrm{~m} / \mathrm{s}$, las pérdidas de presión en la ductería se estiman en $370 \mathrm{~Pa}$ (ASHRAE, 2010), lo que se puede suplir con dos abanicos de flujo axial con una potencia nominal de $10 \mathrm{~kW}$. Se asume que su eficiencia hidráulica es de $40 \%$ (Eck, 1979) y queda un margen adicional de aproximadamente $23 \%$ para suplir las pérdidas adicionales que pueda producir la maqueta y el tramo rugoso de preparación de capa límite o el contraflujo de los abanicos secundarios.

Cualquier abanico industrial que cumpla con los requerimientos simultáneos de caudal (que será proporción directa al área de la sección de trabajo seleccionada para el túnel y a la velocidad máxima de flujo requerida) y de presión (que depende de la geometría de los ductos) es aceptable si los niveles de ruido asociados son permisibles para la localidad de instalación.

Los abanicos secundarios que generan los vórtices variables también son de aplicación industrial, y se trataría de abanicos centrífugos destinados originalmente a servir de cortina de aire: su ancho es varias veces mayor que su diámetro, lo que los hace compactos e inyectarán el caudal con cierta uniformidad a lo largo de su ancho (Eck, 1979) dado que las aspas van en paralelo al eje de rotación del rotor. Adicionalmente, dado que su función es proveer un flujo cortante para iniciar el vórtice, y no el aportar caudal significativo, no es necesario que su capacidad o potencia individual sea comparable a la de los abanicos principales. Para un aporte de aproximadamente $10 \%$ del caudal nominal del túnel, que es de $14,4 \mathrm{~m}^{3} / \mathrm{s}$, se proponen seis ventiladores 
con una ganancia de presión de 500 Pa y un caudal individual de $0,24 \mathrm{~m}^{3} / \mathrm{s}$. Esto correspondería a ventiladores secundarios con una potencia de $400 \mathrm{~W}$ cada uno asumiendo una eficiencia hidráulica de $60 \%$ (Eck) y una holgura de seguridad de $50 \%$ por efectos de sistema imprevistos (ASHRAE, 2013) dada su potencial configuración en contraflujo.

Como se trata de una configuración novedosa, hay incertidumbre en cuanto al ámbito de diámetros de columna de vórtice que podrán generarse con este sistema en función de la velocidad de flujo principal. Dada la complejidad de la condición de flujo quedará para estudios en conjuntos prototipo la caracterización de estas variables previo al diseño final del ensamble.

\section{GRUPOS DE INSTRUMENTACIÓN PRIMARIOS}

La instrumentación necesaria para hacer pruebas en un túnel de viento es altamente especializada en vista de que depende de la información que se desea obtener de la condición de flujo en la sección de pruebas, por lo que no es posible seleccionar un conjunto completo de instrumentación sin incurrir en costos muy elevados. Dicho esto, hay una serie de equipos clave que son comunes a las pruebas que se han mencionado en este documento, necesarios para la ejecución de prácticas de laboratorio, demostraciones, e investigaciones cualitativas. Se propone un conjunto de instrumentos similar al de la Tabla 1.

En el transcurso de los primeros años de funcionamiento, se podrán adquirir equipos más especializados conforme evolucionen los servicios que cubra el túnel de viento.

\section{CONCLUSIONES}

La configuración del ensamble y la selección de los equipos que formarían el concepto propuesto esencialmente obedecen a criterios y métodos ampliamente conocidos por la comunidad técnica asociada al tema de pruebas en túnel de viento. Sin embargo, hay algunos aspectos medulares a la configuración propuesta que merecen enfatizarse dado que tienen impacto directo en la valoración de la viabilidad del proyecto y, sobre todo, en el estímulo del desarrollo de tecnologías autóctonas:

- Se presenta un concepto de túnel de viento con amplias aplicaciones, pero de tamaño, requerimientos y costos considerablemente menores a los de un túnel de viento subsónico para aplicación general. Esto hace atractivo el proyecto a la comunidad científica en Costa Rica en el contexto económico y tecnológico en el que se encuentra.

- Se propone una técnica novedosa para la generación activa y controlable de vórtices verticales para darle mayor versatilidad al túnel de viento de capa límite. La adición de un grupo simétrico de ventiladores secundarios que a los que se les puede variar la velocidad de operación y el ángulo de incidencia en el tramo previo a la sección de pruebas permitirá modelar condiciones más variadas de turbulencia y velocidad de viento local sobre modelos de edificaciones o terrenos. Con esta configuración, la vorticidad y la velocidad de flujo media son parámetros que pueden variarse independientemente; en la mayoría de túneles de viento contemporáneos, esto no es posible sin cambiar la geometría del interior de las paredes de la sección de pruebas.

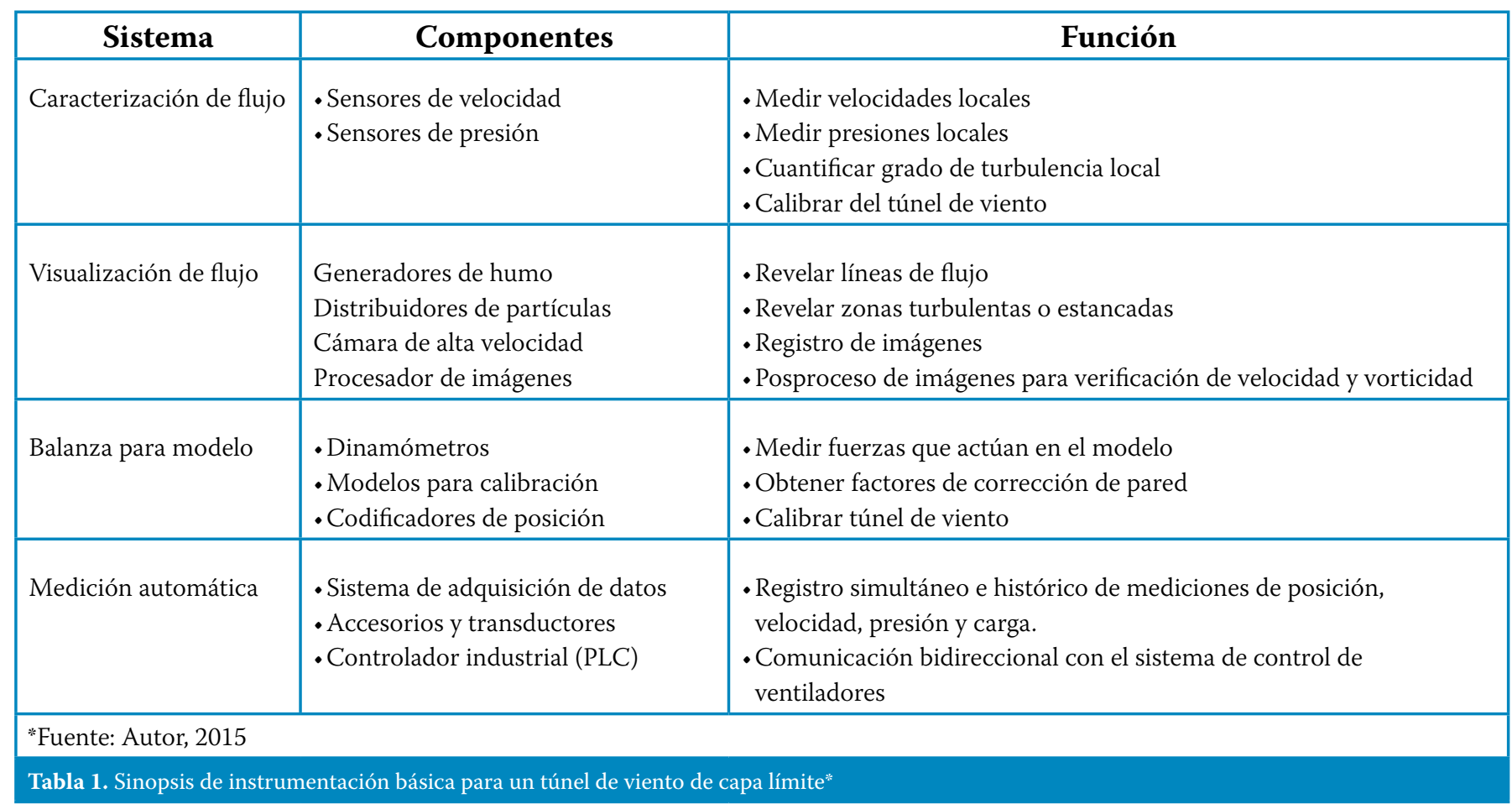


- Las proporciones y dimensiones propuestas se han elegido específicamente para que pueda evolucionar; la longitud de la ductería, el diámetro de los abanicos, y el área de piso pueden permanecer constantes según se requiera y existan recursos, esto para aumentar la máxima velocidad de flujo en la sección de trabajo o agregar componentes que alteren el grado de turbulencia en los distintos tramos.

- Como el equipo requerirá una etapa de prototipado, calibración y puesta en marcha, estimulará a nivel local no solo la ejecución del proyecto inicial, sino que compromete a los operadores a que den prioridad al desarrollo de sus propios instrumentos y métodos en lugar de la compra de equipos de costos innecesariamente altos.

Se espera que este tipo de proyectos contribuyan con el desarrollo regional de los equipos y métodos de determinación precisa de las cargas de viento, modos de vibración de estructuras y valoración en condiciones controladas del impacto que puede tener el viento atmosférico en localidades de interés.

\section{REFERENCIAS}

Ahmad, K., Khare, M., \& Chaudry, K. K. (2005, Setiembre). Wind tunnel simulation studies on dispersion at urban street canyons and intersections. Journal of Wind Engineering and Industrial Aerodynamics, 93 (9), 697-717.

American Society of Heating, Refrigerating and Air-Conditioning Engineers. (2010) Fundamentals. Atlanta: ASHRAE Press.

American Society of Heating, Refrigerating and Air-Conditioning Engineers. (2013) Systems and Applications. Atlanta: ASHRAE Press.

Anina, S., Rüdiger, H., \& Stanko, B. (2015, Setiembre). Numerical simulations and experimental validations of force coefficients and flutter derivatives of a bridge deck. Journal of Wind Engineering and Industrial Aerodynamics, 144, 172-182.

Barlow, J. B., Rae, W. H. R., \& Pope, A. (1999). Low-speed Wind Tunnel Testing (3a ed.). Nueva York: Wiley Interscience.

Bernstein, J. R., S. (1976, Marzo). Reduction of wind-tunnel wall interference by controlled wall flow. NASA Technical Report (CR-2654), 57 pp.

Bianco, L., Wilczak, J., \& White, A. (2008, Agosto). Convective boundary layer depth estimation from wind profilers: Statistical comparison between an automated algorithm and expert estimations. Journal of Atmospheric and Oceanic Technology, 25 (8), 1397-1413.

Colman-Lerner, J. (2011). Wind Tunnels and Experimental Fluid Dynamics Research. Rijeka: InTech.

Colegio Federado de Ingenieros y de Arquitectos de Costa Rica. (2010). Código sísmico de Costa Rica. San José: CFIA.

Eck, B. (1973). Fans: Design and Operation of Centrifugal, Axial-flow and Cross-flow Fans. Oxford: Pergamon Press.

Goodrich, M., \& Gorham, J. (2008). Wind Tunnels of the Western Hemisphere. Washington, D.C.: Federal Research Division. Library of Congress.
Hangan, H. (2015, Agosto). Novel techniques in wind engineering. Conferencia inaugural del 14th International Conference on Wind Engineering. Ontario.

Hirsch, C. (2007). Numerical Computation of Internal and External Flows. Amsterdam: Butterworth-Heinemann.

Kingsbury, N. R. (1990). World directory of aerospace vehicle research and development. Washington, D.C.: GAO-USAF. (Documento interno disponible al público)

Knight, I. (2001, Enero). The design and construction of a vertical wind tunnel for the study of untethered firebrands in flight. Fire Technology, $37(1), 87-100$.

Kundu, P. K., Cohen, I. M., \& Dowling, D. R. (2011). Fluid Mechanics (5a ed.). Amsterdam: Academic Press.

Mehta, R. D., \& Bradshaw, P. (1979, Noviembre).Design rules for small low speed wind tunnels. The Aeronautical Journal of the Royal Aeronautical Society, 443-449.

Monge, J. G. (2010, Noviembre). Túnel de viento de capa límite. Ponencia presentada en el XXIX Minicongreso del Centro de Investigaciones Geofisicas. San José.

American Society of Civil Engineering, (2013). Minimum design loads for buildings and other structures (ASCE 7-10). Reston: ASCE.

Pegg, R. J. (1995). Low speed wind tunnel tests of two waverider configuration models. Sixth International Aerospace, Planes and Hypersonics Technologies Conference. (Descargado el 12 de diciembre de 2005 de http://larc.nasa.gov.com/resources/ 6APC/art.asp)

Pereira, J. (2013). Wind Tunnels: Aerodynamics, Models and Experiments. Nueva York: Nova Science Publichers, Inc.

Reinhold, T. (1982). Wind Tunnel Modeling for Civil Engineering Applications. Cambridge: Cambridge University Press.

Rivera, R. (2005). Diseño y construcción de un túnel de viento para la Facultad de Ingeniería. Tesis de grado para optar por el título de Licenciatura en Ingeniería Civil. San José: Universidad de Costa Rica.

Roberts, S. (2012). Wind Wizard: Alan G. Davenport and the Art of Wind Engineering. Princeton: Princeton University Press.

Schlichting, H., \& Gersten, K. (2000). Boundary-layer Theory (8a ed.). Berlin: Springer.

Shao, Y. (2008). Physics and Modelling of Wind Erosion (2a ed.). Berlin: Springer.

Shojaee, S., Uzol, O., \& Kurç, C. (2014, Febrero). Atmospheric boundary layer simulation in a short wind tunnel. International Journal of Environmental Science and Technology, 11 (1), 59-68. 